\title{
Observations of the 1.35-cm Water-Vapor Line in Venus
}

\author{
W. J. Welch \\ The University of California, Berkeley, Calif.
}

The Venus microwave spectrum has been measured with a $10-\mathrm{ft}$ antenna and scanning radiometer over the frequency range 18 to $36 \mathrm{Mc} / \mathrm{s}$. No evidence was found for the presence of the water-vapor line at $1.35-\mathrm{cm}$ wavelength. Furthermore, comparison with results obtained at MIT, suggest that the spectrum of Venus in this wavelength region may be variable. A large number of theoretical models have been studied, none of which matches the observed spectrum precisely.

(Paper 69D12-599)

\section{Observations of Mars at 12.5-cm Wavelength}

\section{O. Muhleman and T. Sato}

Jet Propulsion Laboratory, California Institute of Technology, Pasadena, Calif.

Observations of Mars at a wavelength of $12.5 \mathrm{~cm}$ have been made with the $85-\mathrm{ft}$ telescope at the Venus site of the Goldstone Tracking Station of the Jet Propulsion Laboratory. The equivalent blackbody disk temperature of the planet was measured on various dates as follows:

$\begin{array}{ll}\text { March 24, 1965 } & 249 \pm 75^{\circ} \mathrm{K} \\ \text { April 6, 1965 } & 199 \pm 57^{\circ} \mathrm{K} \\ \text { April 14, 1965 } & 244 \pm 71^{\circ} \mathrm{K} .\end{array}$

The mean value observed was $225 \pm 39^{\circ}{ }^{\circ} \mathrm{K}$.

(Paper 69D12-600)

\section{On the Nature of the Cloud Layer of Venus (From Radiometric Observations at Microwaves)}

\author{
A. E. Basharinov and B. G. Kutuza \\ Institute for Radio Techniques and Electronics, Moscow, USSR
}

\begin{abstract}
The hypothetic presence of supercooled water drops in the cloud layer of Venus has been checked by extrapolating the $8-\mathrm{mm}$ wave absorption value, obtained from phase variation of radio brightness temperatures, into millimeter and centimeter wave ranges.

It has been shown that the extrapolated values of the radio brightness temperature spectrum are in satisfactory agreement with the radio brightness temperature values measured at the nocturnal side of Venus.

The content of water in the cloud layer is estimated to be about $0.2-0.3 \mathrm{~g} / \mathrm{cm}^{2}$, the absorption in the layer not exceeding 1.5 decibels in the centimeter range and being not above 5 decibels in the millimeter range for a wavelength of above $3 \mathrm{~mm}$.
\end{abstract}


The nature of the cloud layer of Venus was discussed in Lyot [1927; Barrett, 1961; Sinton, 1962; Moroz, 1963; Salomonovich, 1964; Barrett, 1964; Kuzmin, 1964; Pollack and Sagan, 1965] while interpreting optical observations and radio brightness temperature data obtained for the nocturnal side of the planet.

There being a discrepancy between the observed data and those obtained by calculation, the supposed nature of these clouds as consisting of water drops was not confirmed in the cited papers.

In Salomonovich [1964] an analysis of the radio brightness temperature spectrum of Venus has led to the suggestion that the clouds may be formed by drops of hydrocarbon compounds whose dipole molecules have a relaxation time of about $3.5 \times 10^{-12}$ sec.

Detailed data on radiation from Venus within the superhigh frequency range obtained in 1963-1964 and the refinement of knowledge on the water-drop absorption spectra afford the possibility for additional conclusions on the nature of Venus clouds.

Further discussion is based on such a model of the atmosphere of Venus which includes a hot surface and a "cold" cloud layer which is supposed to have a temperature ranging within $270 \pm 30^{\circ} \mathrm{K}$.

According to Kuzmin [1964] and Lyot [1927] the observational data on the phase variation of the radio brightness temperatures of Venus at $10-\mathrm{cm}, 3.2-\mathrm{cm}$, and $0.8-\mathrm{cm}$ wavelengths $\mathrm{can}$ be represented as follows:

$$
\begin{aligned}
& T_{b r}(10)=622+41 \cos \left(\phi \pm 21^{\circ}\right) \\
& T_{b r}(3.2)=621+73 \cos \left(\phi \pm 17.7^{\circ}\right) \\
& T_{b r}(0.8)=427+41 \cos \left(\phi-21^{\circ}\right) .
\end{aligned}
$$

The mean values of radio brightness temperatures for $3.2-\mathrm{cm}$ and $10-\mathrm{cm}$ wavelengths lying close together, it can be supposed that atmospheric absorption is low in this region of the spectrum and thereby the radio brightness temperature at the surface of the planet can be estimated.

In accordance with (1) the radio brightness temperature value averaged over the disk is put at $548{ }^{\circ} \mathrm{K}$ for the nonilluminated side of the planet, and at $694{ }^{\circ} \mathrm{K}$ for the illuminated side.

The difference in the amplitude of radio brightness temperature oscillations at $3.2-\mathrm{cm}$ and $0.8-\mathrm{cm}$ wavelengths is used to determine the atmospheric absorption at the $0.8-\mathrm{cm}$ wavelength.

Taking into account the above-mentioned, the ratio of oscillation amplitudes at $3.2-\mathrm{cm}$ and $0.8-\mathrm{cm}$ wavelengths can be represented as follows:

$$
\begin{array}{r}
\frac{\Delta T_{b r}(0.8)}{\Delta T_{b r}(3.2)}=\frac{\left(T_{b r . i l}-T_{b r . n)}\right) \int_{0}^{\pi / 2} e^{-\frac{\tau(0.8)}{\cos \varphi}} \cos \varphi \sin \varphi d \varphi}{\left(T_{b r . i l}-T_{b r . n)}\right) \int_{0}^{\pi / 2} e^{-\frac{\tau(3.2)}{\cos \varphi} \cos \varphi \sin \varphi d \varphi}} \\
\simeq 2 E[\tau(0.8)]
\end{array}
$$

Here $T_{b r . i l .}$ is the disk-averaged value of radio bright- ness temperature for the illuminated side of Venus; $T_{b r . n}$, is the respective value for the nocturnal side of Venus; $E_{(\tau)}=\int_{0}^{1} e^{-\frac{\tau}{x}} x d x$ is the tabulated function of the integral absorption $\tau$. The value of the integral absorption at the $0.8-\mathrm{cm}$ wavelength is defined, according to $(2)$, as

$$
\tau(0.8)=\arg E\left[\frac{1}{2} \frac{\Delta T(0.8)}{\Delta T(3.2)}\right]
$$

Substituting in (3) the values of radio brightness temperature variation amplitudes obtained from observation data on phase variation (1) we shall have the value of integral absorption $\tau(0.8)=0.32$.

This estimate of integral absorption for the 8-mm wavelength is extrapolated within the microwave range on the assumption that the absorption depends on a cloud layer consisting of water drops.

If the water-vapor pressure in the layer is supposed not to exceed 5 mbar and no allowance is made for the contribution due to selective absorptions, the following relation can be used for extrapolation:

$$
\begin{gathered}
\tau(\lambda)=\tau\left(\lambda_{0}\right) \frac{c(\lambda)}{c\left(\lambda_{0}\right)} \frac{\lambda_{0}}{\lambda} \\
\tau\left(\lambda_{0}\right)=\frac{c\left(\lambda_{0}\right)}{\lambda_{0}} \int_{H_{1}}^{H_{2}} W d l
\end{gathered}
$$

where $W$ is the volume content of drop water; $H_{1}$ and $H_{2}$ are the heights of the upper and lower cloud edges, respectively;

$$
c(\lambda)=\frac{\epsilon^{\prime \prime}(\lambda)}{\left[\epsilon^{\prime}(\lambda)+2\right]^{2}+\left[\epsilon^{\prime \prime}(\lambda)\right]^{2}} .
$$

The real $\epsilon^{\prime}(\lambda)$ and the imaginary $\epsilon^{\prime \prime}(\lambda)$ part of the dielectric constant are known to be defined by

$$
\epsilon^{\prime}(\lambda)=\epsilon_{\infty}+\frac{\epsilon_{0}-e_{\infty}}{1+y^{2}}, \epsilon^{\prime \prime}(\lambda)=\frac{\epsilon_{0}-\epsilon_{\infty}}{1+y^{2}} y
$$

where $\epsilon_{\infty}$ is the value of the dielectric constant in the region of $\lambda \ll c \tau_{r} ; \epsilon_{0}$ is the value of the dielectric constant in the zero-frequency region; $y=2 \pi \frac{c}{\lambda} \tau_{r}\left(\frac{\epsilon_{0}+2}{\epsilon_{\infty}+2}\right)$;
$\tau_{r}$ is the relaxation constant.

According to the data given by Moroz [1963] for temperatures above the melting point, the constant $\epsilon_{\infty}$ is practically independent of the temperature, whereas $\epsilon_{0}$ and $\tau_{r}$ vary essentially with the temperature.

The values of Debye constants in the supercooled region have been obtained by extrapolating the available data into the temperature range below the melting point.

Presented in table 1 are the values of Debye constants from Moroz [1963] for the temperature range above the melting point and those extrapolated into the region below the melting point. 
TABLE 1

\begin{tabular}{c|c|c|c|c|c|c|c}
\hline \hline$C$ & +20 & +18 & +10 & 0 & -5 & -10 & -20 \\
\hline$\epsilon_{0}$ & 80 & 87 & 84 & 88 & 90 & 92 & 96 \\
$2 \pi c \tau_{r}\left(\frac{\epsilon_{0}+2}{\epsilon_{x}+2}\right)$ & 1.53 & 1.66 & 2.24 & 3.59 & 4.36 & 5.39 & 7.40 \\
$\tau_{p(\mathrm{sec})}$ & $7.4 .10^{-13}$ & $8.10^{-13}$ & $1.04 .10^{-12}$ & $1.6 .10^{-12}$ & $1.95 .10^{-12}$ & $24.10^{-12}$ & $3.4 .10^{-12}$ \\
\hline
\end{tabular}

The extrapolation results were checked by the radiometric measurements of absorption in the clouds of the Earth's atmosphere at wavelengths of $4 \mathrm{~mm}, 8 \mathrm{~mm}$, $1.6 \mathrm{~cm}$ and $3.2 \mathrm{~cm}$.

The preliminary experimental estimates of the relative absorptions with allowance for the measurement error are in agreement with the absorption values calculated from (4) and using the values given in table 1.

To give an example, table 2 gives the calculated values of absorption for $0.4-\mathrm{cm}$ and $1.6-\mathrm{cm}$ wavelengths relative to the $0.8-\mathrm{cm}$ wavelength absorption against the respective experimental data for a cloud layer at $-5^{\circ} \mathrm{C}$.

TABLE 2

\begin{tabular}{c|l|l}
\hline \hline$\lambda / \lambda_{0}$ & $0.4 / 0.8$ & $1.6 / 0,8$ \\
$K\left(\lambda, \lambda_{0}\right)$ & 2.7 & 3.2 \\
$K^{*}\left(\lambda, \lambda_{0}\right)$ & 2.4 & 3.3 \\
\hline
\end{tabular}

$K\left(\lambda, \lambda_{0}\right)=\frac{c(\lambda)}{c\left(\lambda_{0}\right)} \frac{\lambda_{0}}{\lambda}-$ calculated values of relative absorptions

$K^{*}\left(\lambda, \lambda_{0}\right)=\frac{\tau(\lambda)}{\tau\left(\lambda_{0}\right)}$-experimental values of relative absorptions

Presented in figure 1 is the absorption spectrum for the cloud layer of Venus obtained by extrapolating by means of (4) the absorption values at 0.8 -cm wavelength on the assumption that the temperature of the layer is $260{ }^{\circ} \mathrm{K}$; for the sake of comparison figure 1

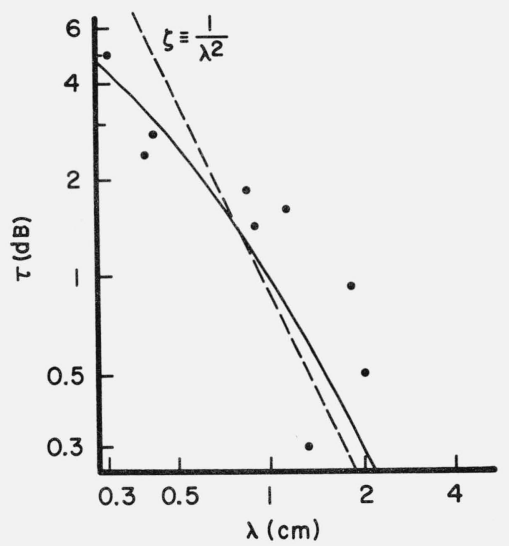

FIGURE 1. Absorption spectrum for the cloud layer on Venus. contains also the estimates of integral absorption obtained from measurement data on the radio brightness temperature of the nocturnal side of Venus for the constants corresponding to the chosen model.

The departure of the absorption estimates is not above $1 \mathrm{~dB}$.

The estimates of the absorption layer temperature in the atmosphere of Venus can be refined by comparing the computed and experimental values of the radio brightness temperature spectrum on the nocturnal side of Venus. For the calculation of the radio brightness temperature spectrum we can use the radiation transfer relation in the form

$$
T_{b r}(\lambda)=T_{b r . n} 2 E\left[\tau\left(\lambda, T_{0}\right)\right]+T_{0}\left\{1-2 E\left[\tau\left(\lambda, T_{0}\right)\right]\right\}
$$

where $T_{b r . n}$ is the disk-averaged value of radio brightness temperature on the nocturnal side of Venus, $T_{0}$ is the temperature of the isothermal absorbing layer, $E\left[\tau\left(\lambda, T_{0}\right)\right]$ is an integral function, see (2). The dependence of the form of the integral absorption function on the temperature of the absorbing layer can serve to evaluate the layer temperature from the condition of agreement between computed and experimental data.

Thus, figure 2 shows that the agreement is satisfactory if a water-drop model and a temperature of $250-260{ }^{\circ} \mathrm{K}$ is assumed for the absorbing layer. On the other hand, the computed values of radio brightness temperature deviate markedly from the experimental ones if the layer temperature of the model exceeds $270{ }^{\circ} \mathrm{K} .{ }^{1}$

From the obtained data on the value of integral absorption the content of drop water in the atmosphere of Venus can be estimated. According to (5),

$$
W_{\Sigma}=\int_{H_{1}}^{H_{2}} W d l=\frac{\tau(\lambda) \lambda}{c(\lambda)}
$$

' It may be remarked that under conditions of supercooling, when the layer temperature is about $260{ }^{\circ} \mathrm{K}$, the extrapolated value of the relaxation constant of the water drops is $2.5 \cdot 10^{-12} \mathrm{sec}$, which does not contradict the conclusion on the expected cloud-layer constants made in (10)

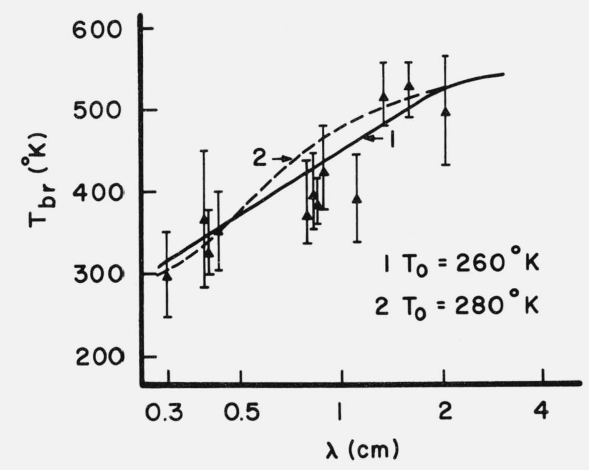

FiguRE 2. The spectrum of radio brightness temperature. 
Substituting in (10) the values of $\tau(\lambda)$ from figure 1 we evaluate the drop-water content as $w$ grams per square $\mathrm{cm}=0.2-0.3 \mathrm{~g} / \mathrm{cm}^{2}$; in the case of clouds with a water content of up to $1 \mathrm{~g}$ per $\mathrm{m}^{3}$ this corresponds to a layer $3 \mathrm{~km}$ thick.

The expected correction to this water-content estimate due to cloud layers of crystalline structure is not higher than 20 to 30 percent.

Of course the data given here are not sufficient for a unique conclusion on the nature of the cloud layer of Venus. The features of the radiation spectrum must be investigated in greater detail; especially, the pattern of phase variation must be studied all over the superhigh frequency range.

\section{References}

Barrett, A. H. (1961), Microwave absorption and emission in the atmosphere of Venus, Astron. J. 133, No. 1, 281-293.

Barrett, A. H. (1964), Radio observations of Venus and the interpretations, Space Sci. Rev. 3, No. 1, 109-135.

Basharinov, A. E., Yu. N. Vetukhnovskaya, A. D. Kuzmin, B. G. Kutuza, and A. E. Salomonovich (1964), Measurements of the brightness temperature of Venus at $8 \mathrm{~mm}$, Soviet Astronomy A. J. 41, No. 4, 707-710.

Drake, F. D. (1964), Microwave observations of Venus 1962-1963, Astron. J. 69, No. 1, 62-64.

Kerr, D. E. (1949), Propagation of Short Radio Waves, lst ed. (McGraw-Hill Book Co., Inc., New York, N.Y.).

Kuzmin, A. D. (1964), Radiophysics 8, No. 6.

Lyot, B. (1927), Annal. Obs. Men. 88, 66.

Moroz, V. I. (1963), Astron. J. 40, 144.

Pollack, J., C. Sagan (1965), The microwave phase effect of Venus, preprint.

Salomonovich, A. E. (1964), Radiophysics 7, No. 1, 51.

Sinton, W. (1962), Comp. on Planet. Atmospher., N.Y.

(Paper 69D12-601)

\title{
An Analysis of Microwave Observations of Venus
}

\section{Carl Sagan}

\author{
Harvard University and Smithsonian Astrophysical Observatory, Cambridge, Mass. \\ and \\ James B. Pollack
}

Smithsonian Astrophysical Observatory, Cambridge, Mass.

The observed variation of the microwave brightness temperatures as a function of wavelength and of phase angle is in good agreement with the simultaneous solution of the one-dimensional heat conduction equation and the equation of radiative transfer. The constancy of the brightness temperature at a given wavelength for consecutive inferior conjunctions argues for an obliquity of the planetary axis of rotation $\leqslant 8^{\circ}$. The phase effect and radar data is consistent with a wide variety of powdered oxides, carbonates, and silicates as predominant constituents of the surface of Venus, but is inconsistent with other materialsin particular, the hypothesized polycyclic aromatic hydrocarbons. The phase data points uniquely to retrograde rotation and to a small surface phase lag.

With the deduced subsurface temperature variations the predicted $8-\mathrm{mm}$ phase effect can be estimated under a variety of assumptions and compared with observations. The observed phase effect is in disagreement with that expected from theory if atmospheric water vapor or carbon dioxide were the source of the millimeter-wave opacity; but is in agreement with theory if the opacity is due to dust distributed through the lower atmosphere and preferentially abundant in the illuminated hemisphere. The observations and theory are also in excellent agreement if the millimeter attenuation is due to clouds.
Determination of plausible surface materials properties permits the following estimates to be made of surface thermometric temperatures: mean disk, $700{ }^{\circ} \mathrm{K}$; subsolar point, $1000{ }^{\circ} \mathrm{K}$; antisolar point, $610{ }^{\circ} \mathrm{K}$; pole, $470{ }^{\circ} \mathrm{K}$. These temperatures are also in agreement with previous values obtained by Pollack and Sagan from their analysis of microwave interferometric observations of Venus. The corresponding surface pressures are $\sim 50 \mathrm{~atm}$.

The low radar reflectivity reported at $3.6 \mathrm{~cm}$ cannot be due to a general $3.6-\mathrm{cm}$ absorption by the atmosphere or clouds. This result can be attributed to anomalously high absorption above a surface cold spot near the subterrestrial point at inferior conjunction, or to the variation of porosity, and therefore, of dielectric constant, with depth.

The output of the 19-mm channel of the Mariner II microwave radiometer can also be used to check various models of the atmosphere and clouds of Venus. The observed Venus limb darkening is due both to the variation of surface emissivity with angle, and to the slant path attenuation by microwave absorbers in the atmosphere and clouds. The observations cannot be consistently explained if the microwave attenuation is caused by $\mathrm{CO}_{2}, \mathrm{H}_{2} \mathrm{O}$, or absorbing dust particles continuously distributed throughout the atmosphere of Venus. The limb-darkening observations 